\title{
Peirce's Notion of Diagram Experiment \\ Corrollarial and Theorematical Experiments With Diagrams
}

Frederik Stjernfelt, Aarhus

I hope that, before I cease to be useful in this world, I may be able to define better than I now can what the distinctive essence of theoric thought is. I can at present say this much with some confidence. It is the directing of the attention to a sort of object not explicitly referred to in the enunciation of the problem at hand ... (Peirce 'Specimens of Mathematical Mazes', 1908, NEM III, p. 622)

A central aspect of Peirce's doctrine of diagrammatical reasoning is the idea of using the diagram as a tool for making deductions by performing rulebound experiments on the diagram. This paper discusses Peirce's distinction between two classes of diagram proofs, 'corollarial' and 'theorematical', respectively - a distinction he himself saw as his first major discovery. Theorematical reasoning concerns diagram experimentation involving the introduction of new material - in particular, I shall investigate the issue of the structure of theorematical diagram experiments, propose three types of such experiments and connect them to the role played by hypostatic abstractions in those experiments.

The increasing interest in Peirce's philosophy of logic as well as his philosophy of science highlights the importance of his notion of deductive reasoning as based on diagrams. As argued by several authors in Moore 2010 (Tiercelin and Cooke), this can be taken as providing an original solution of the dilemma which has haunted much philosophy of mathematics since Benacerraf's famous 1973 'Mathematical Truth' article against Platonism (in Benacerraf and Putnam 1983). Benacerraf's paper argued against Platonism and the existence of abstract objects in mathematics by setting up the following dilemma: 
1) Mathematics claims the existence of abstract objects outside of time and space; 2) Acquisition of knowledge takes place by means of a causal process connecting an object with a knowing subject. But as abstract objects are causally inert, we must either accept Platonism and reject causal epistemology - or we must embrace causal epistemology and refuse Platonism. Due to the prominence of causal reference theories at the time, Benacerraf's choice seemed obvious: abstract objects and Platonism must be discarded in the face of the seeming evidence of causal epistemology. Literally taken, Benacerraf's argument would, in fact, eliminate not only abstract objects but a series of other aspects of the world, such as many properties (color, pitch, shape ...) which may also be suspected for being causally inert in the billard-ball causation theory of the second horn of the dilemma.

Causal reference theories hardly hold the attraction which they did in the 1970 s, and the role of diagram experiments in an alternative, Peircean way of cutting the cake is the following. To Peirce, deduction and mathematical reasoning are one and the same. Mathematics is defined by two things, methodologically and substantially, respectively. The former comes from the definition of mathematics that he inherited from his father, the mathematician Benjamin Peirce: mathematics is the science that draws necessary conclusions. Peirce's own addition to this doctrine pertains to the subject matter of those necessities: the object of mathematics is hypotheses concerning the forms of relations. All mathematical knowledge thus has a hypothetical structure: if such and such entities and structures are supposed to exist, then this and that follows. We might call this weaker variant of commitment to abstract objects 'hypothetical Platonism'. This admission liberates you, of course, from the presupposition of a strange, space-time-less realm of real existencebut it commits you, on the other hand, to further existence modes than that of particular individuals, which is why diehard nominalists will hardly feel attracted by Peirce's alternative. Peirce's doctrine operates with no less than two further existence modes than that of individuals, namely that of possibility - 'May-Bes' - and that of real possibility - 'Would-Bes'. Mathematics being hypothetical through-and-through then forms a subset of the latter. The crucial role of diagrams, now, is that the notion of reasoning by diagram experiments furnishes an epistemological alternative to Benaceraffian causal reference. The idea is that diagrams form the epistemological means of accessing hypothetical abstract objects. They do that in two steps, as it were. One step is taking a diagram token, a drawing on paper, blackboard, computer screen, 
or in the imagination, and subjecting it to 'prescission', the imaginary stripping it of accidental qualities so that only the relevant, controllable, general, schematic relations are left-permissing the observer to grasp, through the token, its type. This process of prescission, of course, is not arbitrary nor subjective and is governed by symbols and rules, explicitly or implicitly. Once the type is grasped, it may, by the intermediary of its physical token, be subjected to experimental manipulation, in imagination or on a physical diagram replica, or both. Certain types of transformation are allowed, others not so, corresponding to truth-preserving logical reasoning steps. So diagram experimentation constitutes the if-then hypothetical structure of mathematics and thus gives mathematical knowledge its conditional, modal character.

The observation of diagram tokens/types, of course, is prefigured in the perception of ordinary objects as tokens of types - just like the prescission process stripping the token of its accidental qualities in order to access its type is a more formalized version of similar processes when we address natural kinds by stripping away accidental properties in order to constitute categories like red, chairs, running - or even the category of an individual persisting in time despite its changing appearances. General structures and shapes of reality are present already in the perceptual stream, and it is no wonder we, as biological beings, have become adapted to focus upon such features in perceptual structures. This very ability, however, may now be recycled apart from its basis in real objects to be put to use to purified imaginary objects like those of mathematics.

This argument pertains to pure, mathematical diagram reasoning; now what about the vast amount of applied diagrams representing empirical states-of-affairs? Peirce's system of the sciences offers an explanation of the efficacy of such diagrams - namely that they inherit, explicitly or implicitly, the mathematical structure of pure diagrams and add further constraints to those diagrams stemming from the special science of the domain to which they pertain. ${ }^{1}$ Thus, all deductive reasoning, everyday or scientific, is taken to involve a mathematical-diagrammatical scaffolding, and necessary inferences in all sciences as well as in everyday reasoning employ mathematics, implicitly or explicitly. In Stjernfelt (2007), I have attempted a reconstruction of Peirce's overall doctrine of diagrams and diagrammatical reasoning, arguing that this cluster of ideas forms the center of a Peircean epistemology as well as it constitutes an important contribution to contemporary realist semiotics in general. 
In this paper, I shall take a closer look at the notion of diagram experiment based on Peirce's famous distinction between two such classes of experiments, giving rise to Corollarial and Theorematical reasoning, respectively. On the base of this distinction, a series of important issues are addressed. To what degree does this distinction capture different formal classes of problem difficulty? How may we distinguish between different types of Theorematic reasoning? And what is the relation between diagram experiments and hypostatic abstraction?

In the years after 1900, Peirce returns over and over again to the Corollarial/Theorematical-distinction, famously celebrating it as his own first "real discovery" (in his Carnegie application 1902). The overall idea is that corollarial deduction has a conclusion which fleshes out a proposition which lay already implicitly in the premisses - thus conforming to the Kantian idea of logical conclusions offering nothing which was not already defined in the premisses. By contrast, theorematic (or theorematogenic, or theoretic, or theoric) reasoning forms a more demanding and creative type of reasoning where some new elements must be experimentally added to the premisses before reaching the conclusion.

Take a simple example: asking the question of the size of the perimeter of a square with the side $s$, the conclusion may be reached based on the very definition of a square as a quadrangle with four equal sides - or by a very simple diagram experiment of counting sides, using the following diagram:

Fig.1: Square with the side $s$

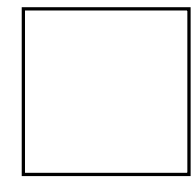

The result of $4 s$ is easily reached by both of these means. By comparison, the famous Euclidean proof of the angle sum of the triangle being equal to two right angles may serve as an example of theorematic reasoning:

Fig. 2: Angle sum of a triangle

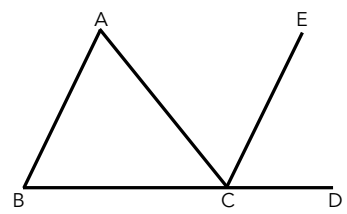


This proof requires the addition of auxiliary lines to the triangle- here CE and $\mathrm{CD}$, parallel to $\mathrm{AB}$ and prolonging $\mathrm{BC}$, respectively - to establish the proof based on the fact that the three angles meeting at $\mathrm{C}$ have the same sizes as those of the triangle. BCA participates in both of the two sums, $\mathrm{ACE}$ is equivalent to $\mathrm{BAC}$, while $\mathrm{ECD}$ is equivalent to $\mathrm{ABC}$. ${ }^{2}$ The sum of the three angles meeting at $\mathrm{C}-\mathrm{BCA}, \mathrm{ACE}$, and $\mathrm{ECD}$ - is obviously two right angles. But this is impossible to derive from mere definitions of 'angle' and 'triangle' just like it is impossible to prove from the triangle diagram without any additions.

The terminology of corollarial/theorematic comes from Euclid whose editors named simple inferences corollaries - while propositions to be proved were theorems. Peirce judges that some of Euclid's theorems are, in fact, mere corollaries - the overall distinction is taken to rely upon the necessity of experimenting by adding new elements to the diagram, elements which disappear in the final, general statement of the proof. The theorem that the angle sum of a triangle equals two right angles does not, for example, in any way refer to the subsidiary lines necessary to reach the proof.

The basic issue behind this distinction is, of course, the duplicity of mathematics, being apodictic and inexhaustible at one and the same time. How is it possible that mathematicians find results by necessity while at the same time new, unexpected discoveries abound among these results, just like in the empirical sciences? This issue troubled Peirce for a long time. Already when constructing his first formal language for logic, in his 1885 masterpiece 'On the Algebra of Logic', he reasoned:

It has long been a puzzle how it could be that, on the one hand, mathematics is purely deductive in its nature, and draws its conclusions apodictically, while on the other hand, it presents as rich and apparently unending a series of surprising discoveries as any observational science. Various have been the attempts to solve the paradox by breaking down one or other of these assertions, but without success. The truth, however, appears to be that all deductive reasoning, even simple syllogism, involves an element of observation; namely, deduction consists in constructing an icon or diagram the relations of whose parts shall present a complete analogy with those of the parts of the object of reasoning, of experimenting upon this image in the imagination, and of observing the result so as to discover unnoticed and hidden relations among the parts. (CP 3.363) 
Here, the inexhaustibility of mathematics is explained by means of Peirce's first, germ-like diagram reasoning doctrine - as a generalization, interestingly, of his linear algebra of logic, far from the ordinary conception of diagrams. The 'unnoticed and hidden' relations obtainable by diagram observation, of course, are what are later taken to require theorematic deduction, in addition to mere inference from definitions.

Even if the problem addressed by the distinction is thus an early concern in Peirce, it seems only in the years after 1900 that he explicitly coins the corollarial/theorematic distinction and sets out to elaborate it. Only in 1901, we seem to witness the nascent terminology of the distinction appearing:

\section{Oct 12}

If my present view, held for four or five years, is right that Abduction Deduction Induction are Premarian, Secundarian, and Tertian, then there ought to be two types of Deduction \& three of Induction [...] Now I don't recognize any such two types of Deduction. [...] We can distinguish Deductions into those which are corollarific and those which are theorematogeneous. The former merely require the careful consideration of the conclusion, the latter involve outside considerations, - subsidiary lines, etc. But this seems a methodeutic not a critical distinction. (Logic Notebook Ms. 339, p. 362; earlier on the page, Peirce attempts to distinguish proposition deductions and term deductions).

Later, Peirce will include the corollarial/theorematic distinction in his critical table of inference types - but this early quote points to the fact that the latter part of the distinction sits uneasily on the critical/methodeutic divide (today, we would rather speak of logic versus heuristics or theory of science). Theorematic reasoning requires an inventiveness or even ingenuity which makes it alien to a narrow concept of logic - even if its results, on the other hand, remain purely deductive.

This apparently simple distinction covers a whole bunch of interesting issues: that of the much more outspoken experimental character of theorematical reasoning as compared to corollarial reasoning, that of finding suitable new elements to add, that of instantiating those elements in particulars (only a particular set of lines in the angle sum example will lead to the proof), that of the character of those elements, that of the relation of the diagram experiment to verbal instructions and definitions, that of the strategy of 
finding the proper elements to add. Probably for this reason, Peirce's description of theorematical reasoning differs to some extent from time to time.

\section{Theorematic diagram experiments in Peirce}

Let us run through Peirce's different definitions.

1) The basic idea is the indirect conception that theorematical reasoning, unlike corollarial reasoning, is not reducible to inferences from concept definitions: 'An accurate definition of Corollarial Demonstration would require a long explanation; but it will suffice to say that it limits itself to considerations already introduced or else involved in the Explication of its conclusion; while Theorematic Demonstration resorts to a more complicated process of thought.' ('A Neglected Argument for the Reality of God', 1908, CP 6.471, EPII, p. 442) This brief definition of theorematic reasoning, of course, is merely negative and contrastive vis-à-vis Kant's description of logic as tautological, and most of Peirce's descriptions of the pair of concepts take their point of departure in the inability of certain theorems to be proved by corollarial reasoning:

Deductions are of two kinds, which I call corollarial and theorematic. The corollarial are those reasonings by which all corollaries and the majority of what is called theorems are deduced; the theorematic are those by which the major theorems are deduced. If you take the thesis of a corollary, i. e., the proposition to be proved, and carefully analyze its meaning, by substituting for each term its definition, you will find that its truth follows. ('On the Logic of Drawing History from Ancient Documents', 1901, CP 7.204, EPII, p. 96 - after which the quote continues with a more positive definition of theorematic reasoning (cf. below)).

Even if corollarial reasoning counts as the ideal and should be preferred whenever possible because of its simplicity, a certain class of 'major theorems' require more than careful description in terms of concept analysis. This necessity stems from the general impossibility of defining things, in all cases, so that all their properties will be corollaries from their definition. Peirce addresses this when claiming that the best translation of Greek 'episteme' is 'comprehension' which is '... the ability to define a thing in such a manner that all its properties shall be corollaries from its definition. Now it may be 
that we shall ultimately be able to do that, say for light or electricity. On the other hand, it may equally turn out that it forever remains as impossible as it certainly is to define number in such a way that Fermat's or Wilson's theorems should be simple corollaries from the definition.' ('On Science and Natural Classes', 1902, EPII, p. 129). Even if much in arithmetics is corollarial (such as Kant's famous $7+5=12$ which Peirce refuses to admit the status of synthetic a priori for the same reason), complicated theorems of arithmetics are not. ${ }^{3}$ So the impossibility of defining things, in all cases, so that all their essential properties flow from the definition, obviously forms the first argument for the necessity of theorematic reasoning.

2) A basic way of describing theorematic reasoning more positively, now, is as involving the addition of new elements to the premisses (abstractions or not, foreign ideas or existential instantiations of general objects the existence of which is granted by the universe of discourse). Peirce seems to have received this idea about the introduction of a new element from no less than George Boole's widow in 1898:

The widow of the great Boole has lately written a little book in which she points out that, in solving a mathematical problem, we usually introduce some part or element into the construction which, when it has served our purpose, is removed. Of that nature is a scale of quantity, together with the apparatus by which it is transported unchanged from one part of the diagram to another, for the purpose of comparing those two parts. Something of this general description seems to be indispensable in mathematics. ('The Logic of Mathematics in Relation to Education', 1898, CP 3.561)

- and his overall development of the corollarial/theorematic distinction now covers the following decade. The addition of such objects is taken to be the subject of an additional lemma to the premisses, supported by a postulate. Continuing the above quote from 'On the Logic ...' (1901), Peirce writes:

But when it comes to proving a major theorem, you will very often find you have need of a lemma, which is a demonstrable proposition about something outside the subject of inquiry; and even if a lemma does not have to be demonstrated, it is necessary to introduce the definition of something which the thesis of the theorem does not contemplate. In the most remarkable cases, this is some abstraction; that is to say, a subject whose existence consists in some fact about 
other things. Such, for example, are operations considered as in themselves subject to operation; lines, which are nothing but descriptions of the motion of a particle, considered as being themselves movable; collections; numbers; and the like. ('On the Logic of Drawing History from Ancient Documents', 1901, CP 7.204, EPII, p. 96).

In Peirce's debatable analysis, lines are abstractions from the trajectory of particles - so the subsidiary lines in the angle sum proof are taken to be examples of the introduction of abstractions. Be that as it may ${ }^{4}$, the quote given here overlooks the important issue of the selection of those lines. The postulate in Euclid that given a line and a point, a line through the point may be drawn which is parallel to the line given, obviously lies behind the lemma of introducing the two particular auxiliary lines in the proof. But not any lines added to the original triangle would lead us to the proof. So the selection of particular objects to add becomes an important issue. Hintikka, in his famous development of Peirce's notion of theorematic reasoning, takes this 'existential instantiation' in the shape of 'witness individuals' to constitute the core of theorematical reasoning, adding further quantified variables to those referred to in the premisses. Sun-Joo Shin (2010) emphasizes the importance of this individualizing step in reasoning: much has been spoken, since the British empiricists, of the access to the triangle in general, but the inverse movement, that of selecting the right individuals to add in a proof, has received much less attention. Hintikka insists that the addition of individuals to the premisses constitutes the very core of Peirce's idea: 'What makes deduction theorematic according to Peirce is that in it we must envisage other individuals than those needed to instantiate the premise of an argument.' (1980, 110)-also other than those needed to express its conclusion, we may add. This is what constitutes the basis of Peirce's 'brilliant insight [...] that this geometrical distinction can be generalized to all deductive reasoning.' (1980, 109). Thus, in Hintikka's reconstruction, '... a valid deductive step is theorematic, if it increases the number of layers of quantifiers in the proposition in question.' (1980, 110). To Hintikka, this solves the ancient Aristotelian riddle of logical incontinence-how can it be that one may fail to grasp the sum total of logical consequences of the amount of knowledge in one's possession. This is because many of those consequences require the theorematic addidion of further individuals for their proof, and Hintikka surmises that the difficulty of a problem is roughly proportional to the amount of new individuals needed for its solution (Hintikka 1980, 113; Stjernfelt 
2007, 107-8). This particularity of auxiliary individuals, much discussed after Hintikka's reinterpretation of theorematical reasoning, is surprisingly rarely addressed in Peirce; however, this late quote connects the basis of the additional elements in a general postulate with the particularity of those elements:

Of my two divisions of Deductions, one is into Corollarial and Theorematic Deduction. The former requires nothing more than a logical analysis of the premisses to furnish the conclusion. The latter involves as one of its premisses a postulate, or proposition asserting the possibility of any object which lies in certain definite general relation to any existing objects of a certain kind. E. g. Between any two points on a line it is possible to place a third. Now to derive from this postulate the particular consequence that will lead to the conclusion requires not merely sagacity or Aristotle's eustokha [...] but also imaginative genius in all its complexity of resources. (Ms. 764, reel 16, 1282-83, late, seemingly 1910-11)

Here, the requirement of imaginative genius - implicitly compared to the laborious teasing out of corollarial definition consequences - is highlighted as required for finding the appropriate particular elements to add. Shin (1997; 2010) also insists upon the importance of selecting the right individuals, among many possible, to conduct the proof. ${ }^{5}$

Other times, it is rather the general or abstract (which is not the same) character of the added elements which is emphasized: 'To the Diagram of the truth of the Premisses something else has to be added, which is usually a mere May-be, and then the conclusion appears.' (letter to James 25. dec 1909, EPII, 502). A May-be, in Peirce's late metaphysics, is a possibility which is, of course, general. Especially when talking about the added elements in this general way, Peirce insists they are foreign to the theorem which the proof intends to establish:

What I call the theorematic reasoning in mathematics consists in so introducing a foreign idea, using it, and finally deducing a conclusion from which it is eliminated. Every such proof rests, however, upon judgments in which the foreign idea is first introduced, and which are simply self-evident. (Carnegie Application (L75), 1902, NEM IV, p. 42). ${ }^{6}$

The question is: are the additional elements particular instantiations selected on the basis of general possibilities granted in the relevant universe of 
discourse (like the subsidiary lines of the angle sum proof granted by Euclid's postulates), or does the addition concern a new, general principle or idea? Judson Webb, in an important paper on Hintikka's philosophy of logic, also points to the fact that, in discussing different proofs of Desargues' theorem, Peirce involves different types of theorematic reasoning: 'There are just two distinct kinds of things we can introduce into a proof that do not appear in such a theorem: auxiliary lines and the idea of length. The former are only new objects of the same kind occurring in the theorem, while the latter is a new concept that is "foreign" to it.' (Webb 2006, 249). Peirce, however, did not seem to pay explicit attention to this important distinction to which we shall return later.

He did, however, introduce another distinction between subtypes of theorematic reasoning. In the famous description of the two kinds of deduction in the Carnegie application, the description in terms of new elements gives rise to a subdivision of theorematic reasoning based on the abstract or non-abstract character of that reasoning:

My first real discovery about mathematical procedure was that there are two kinds of necessary reasoning, which I call the corollarial and the theorematic, because the corollaries affixed to the propositions of Euclid are usually arguments of one kind, while the more important theorems are of the other. The peculiarity of theorematic reasoning is that it considers something not implied at all in the conceptions so far gained, which neither the definition of the object of research nor anything yet known about could of themselves suggest, although they give room for it. Euclid, for example, will add lines to his diagram which are not at all required or suggested by any previous proposition, and which the conclusion that he reaches by this means says nothing about. I show that no considerable advance can be made in thought of any kind without theorematic reasoning. When we come to consider the heuretic part of mathematical procedure, the question how such suggestions are obtained will be the central point of the discussion.

Passing over smaller discoveries, the principal result of my closer studies of it has been the very great part which an operation plays in it which throughout modern times has been taken for nothing better than a proper butt of ridicule. It is the operation of abstraction, in the proper sense of the term, which, for example, converts the proposition "Opium puts people to sleep" into "Opium has a dormitive virtue". This turns out to be so essential to the 
greater strides of mathematical demonstration that it is proper to divide all theorematic reasoning into the non-abstractional and the abstractional. I am able to prove that the most practically important results of mathematics could not in any way be attained without this operation of abstraction. It is therefore necessary for logic to distinguish sharply between good abstraction and bad abstraction. (Carnegie Application (L75), Draft C, 90-102, NEM IV, p. 49).

This distinction between abstractional and non-abstractional theorematical reasoning has been taken up by Stephen Levy and Michael Hoffmann (1997; forthcoming) in their efforts to outline taxonomies of theorematic reasoning. But is it the case that this idea might, simultaneously, constitute a basis for the distinction between theorematic reasoning by means of existential instantiation on the one hand and the introduction of new, foreign ideas on the other? Peirce does not further develop his distinction between abstractional and nonabstractional theorematic reasoning, so it is difficult to decide. Suffice it to say that it is not evident these two distinctions are identical or even co-extensive; the introduction of certain abstract objects may be permitted in the formalism used and in that sense not being new or foreign (just like the introduction of lines in a geometric proof or a variable in an equation) - the foreign idea seems to comprise a special class of abstractions only.

3) An interesting feature of the descriptions in terms of added elements quoted here is that they do not refer to deductions in terms of diagram experiments. Diagram experiment, however, is taken to constitute the center of deduction in general, and of theorematic deduction in particular. In a parallel draft for the Carnegie application, Peirce thus characterizes theorematic reasoning as follows:

Theorematic deduction is deduction in which it is necessary to experiment in the imagination upon the image of the premiss in order from the result of such experiment to make corollarial deductions to the truth of the conclusion. The subdivisions of theorematic deduction are of very high theoretical importance. (Carnegie Application (L75), 1902, NEM IV, p. 38).

The year after, in the masterful Syllabus accompanying his Lowell lectures, Peirce connects the experimental character of theorematic reasoning to the ingenuity required as well as to observation; it '... is one which, having represented the conditions of the conclusion in a diagram, performs an 
ingenious experiment upon the digram, and by the observation of the diagram so modified, ascertains the truth of the conclusion.' (Syllabus, 1903, CP 2.267, EPII, p. 298). In one of the drafts of the Lowell lectures, Peirce connects these two descriptions, now taking the addition of new material to be a subtype of experiment:

I draw a distinction between Corollarial consequences and Theorematic consequences. A corollarial consequence is one the truth of which will become evident simply upon attentive observation of a diagram constructed so as to represent the conditions stated in the conclusion. A theorematic consequence is one which only becomes evident after some experiment has been performed upon the diagram, such as the addition to it of parts not necessarily referred to in the statement of the conclusion. (Lowell Lectures, Ms. 456, (p. 49; ISP 28) transcription by Helmut Pape).

Here, it is not made explicit which other types of experiment there might be besides the addition of new elements. In an early account for diagram experimentation, however, Peirce provides such an example:

Deduction is that mode of reasoning which examines the state of things asserted in the premisses, forms a diagram of that state of things, perceives in the parts of that diagram relations not explicitly mentioned in the premises, satisfies itself by mental experiments upon the diagram that these relations would always subsist, or at least would do so in a certain proportion of cases, and concludes their necessary, or probable, truth. For example, let the premiss be that there are four marked points upon a line which has neither extremity nor furcation. Then, by means of a diagram,

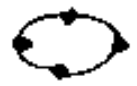

we may conclude that there are two pairs of points such that in passing along the line in any way from one to the other point of either pair, one point of the second pair will be passed an odd number of times and the other point an even (or zero) number of times. This is deduction. (Untitled manuscript, c. 1896, 1.66) ${ }^{7}$

In this example, the diagram experiment is undertaken by following a trajectory along the closed curve, until realizing that each full turn will add 2 to the number of passages of each point pair - so with respect to odd/even, the result will stay the same as the very first half trajectory, passing one point 
1 time, the other point 0 times. This experiment hardly introduces any new ideas at all, but it does involve instantiation, this time of a trajectory moving in the diagram. So the new elements added may also be actions performed on the diagram. In the 'Minute Logic' of 1902, Peirce hints at those other experiment possibilities:

Just now, I wish to point out that after the schema has been constructed according to the precept virtually contained in the thesis, the assertion of the theorem is not evidently true, even for the individual schema; nor will any amount of hard thinking of the philosophers' corollarial kind ever render it evident. Thinking in general terms is not enough. It is necessary that something should be DONE. In geometry, subsidiary lines are drawn. In algebra permissible transformations are made. Thereupon, the faculty of observation is called into play. Some relation between the parts of the schema is remarked. But would this relation subsist in every possible case? Mere corollarial reasoning will sometimes assure us of this. But, generally speaking, it may be necessary to draw distinct schemata to represent alternative possibilities. Theorematic reasoning invariably depends upon experimentation with individual schemata. ('Minute Logic', CP 4.233, c. 1902)

Here, the mere introduction of new elements or ideas as additional general terms is not deemed sufficient - the experiment is supposed to perform an action manipulating the diagram - drawing the auxiliary lines - or, in the algebraical example, undertaking transformation granted by the relevant symbol manipulation rules. In the Euclidean example, transformations including the movement of geometrical objects on the plane permitted (rotations, mirrorings, translations, etc.) obviously form a class of experiments different from those of introducing new elements, just like, in arithmetics, the transformation possibilities given by calculation rules (and more generally, in algebra, symbol manipulation rules), provide such experiment possibilities.

An important issue here - both related to the "addition of new elements or foreign ideas" and to the "experiment" aspects - is the relation between theorematic reasoning and abduction. A finished piece of theorematic reasoning, of course, is deductive - the conclusion follows with necessity from the premisses. But in the course of conducting the experiment, an abductive phase appears when investigating which experimental procedure, among many, to follow; which new elements or foreign ideas to introduce. This may require 
repeated, trial-and-error abductive guessing, until the final structure of the proof is found - maybe after years or centuries. Exactly the fact that neither premisses nor theorem needs to contain any mentioning of the experiment or the introduction of new elements makes the abductive character of experimentation clear. Of course, once the right step has been found, abductive searching may cease and the deductive character of the final proof stands out.

4) A further description of the corollarial/theorematic distinction makes it correspond to reasoning with words or schemata, respectively. The quote just given from the 'Minute Logic' continues with the conclusion that

We shall find that, in the last analysis, the same thing is true of the corollarial reasoning, too; even the Aristotelian "demonstration why." Only in this case, the very words serve as schemata. Accordingly, we may say that corollarial, or "philosophical" reasoning is reasoning with words; while theorematic, or mathematical reasoning proper, is reasoning with specially constructed schemata. ('Minute Logic', CP 4.233, c. 1902).

This complicated claim identifies corollarial reasoning with philosophical reasoning in words (implicitly placing a severe limitation on the powers of such reasoning), as compared to the constructive power of theorematic reasoning using specially constructed schemata and being able to make 'demonstration that'. Immediately, however, words are also taken to constitute such schemata, even if maybe simpler and less directly accessible than 'specially constructed' schemata. The ubiquity of such schemata also outside of science (maps, graphs and tables in newspapers, media, commodities, etc.) points to the fact that the distinction between words and constructed schemata does not, as it might be immediately asumed, coincide with that of everyday reasoning and science. Rather, the idea that conceptual reasoning forms a simple version of schematic reasoning points to the idea of the distinction between corollarial and theorematic as being a gradient continuum rather than two mutually exclusive classes - also supported by the fact that theorematic reasoning examples differ enormously in complexity and the amount of new elements required. We shall return to this below.

5) A final characterization of theorematic reasoning is that of requiring a new point of view of the problem. We find a simple version of this in Ms. 773 (Reel 16, MF 1434-35): 'I spoke of Deduction as the compulsive kind of reasonings. Almost all the theoric inferences are positively creative. That is, 
they create, not existing things, but entia rationis which are quite as real. This blackboard is black. Theoric deduction concludes that the board possesses the quality of blackness and that blackness is a simple object, called an ens rationis because that theoric thought created it.' Here, the hypostatic abstraction from 'black' to 'blackness' is taken as an example of theoric deduction.

Here is a terminological problem. In many cases, 'theoric' is used interchangeably with 'theorematic'; in other contexts, Peirce seems to intend a slightly different meaning by the concept 'theoric'. One of his paradigm examples is that of Desargues' theorem (two triangles which are centrally in perspective are also axially in perspective, usually referred to by Peirce as 'the ten point theorem' $)^{8}$ - a 17. century geometry proof recently rediscovered in Peirce's time by von Staudt in the context of projective geometry. Here, Peirce uses the notion of 'theoric' to refer to the 'new point of view' which may introduce a third dimension to the diagrammatical representation of the 2-d theorem, thereby making it much more immediately graspable than much more cumbersome proofs using lenghts of lines:

Fig. 3

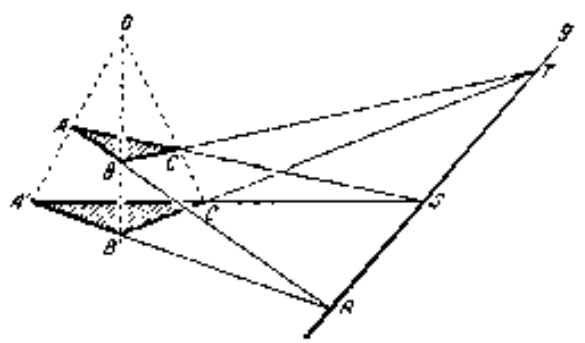

The two triangles lying in a central perspective as seen from the point 0 have the intersections of their sides coinciding on the same line (axial perspective). This figure (Hilbert and Cohn-Vossen, quoted from Hoffmann, forthcoming p. 18) shows how a three-dimensional interpretation of the originally 2-d planar diagram makes it easy to grasp that the two planes of the triangles meet at the line $g$. Peirce returns over and over again to this proof, taking it as a central example of 'theoric' or 'theorematic' reasoning. An alternative proof remains restricted to two dimensions but rests upon another theorematic addition, namely that of the length of lines which is also not mentioned in the original theorem.

Michael Hoffmann has made a strong case that this adoption of a new 
point of view should be called 'theoric', differing from theorematic reasoning because it simply constitutes a gestalt shift in the conception of the problem rather than the necessary experimental introduction of new elements in the deduction process (Hoffmann, forthcoming). Hoffmann's interpretation is based on the use of the term 'theoric' in Ms. 318 and Ms. 754, both of them from 1907. From the large Ms. 318 on pragmatism, Hoffmann quotes the following description of 'theoric' reasoning which consists ' ... in the transformation of the problem, - or its statement, - due to viewing it from another point of view' (ibid., CSP 68 = ISP 225). In the brief Ms. 754 (notes for a 'talk to the Phil. club' April 12, 1907), Peirce writes:

I formerly, quite dubiously, divided Deductions into the Corollarial \& the Theorematic. Explain these. Deduction will better be called Demonstration. But further study leads me to lop off a corollarial part from the Theorematic Deductions, which follows that part that originates a new point of view. This part of the theorematic procedure, I will call theôric reasoning. It is very plainly allied to retroduction, from which it only differs as far as I now see in being indisputable. (Peirce, quoted in: Hoffmann, forthcoming, p. 27, n13) ${ }^{9}$

The core of theorematic reasoning, following this quote, is taken to be the theoric introduction of a new viewpoint - the rest seems to be mere corollarial reasoning. I am not convinced, though, that Peirce, by the introduction of the term 'theoric' in 1907 intends a new concept, completely different from that of 'theorematic'. In the Ms. 754 quote just given - which forms a hapax, the only place, to my knowledge, where Peirce uses both of the notions 'theoric' and 'theorematic' - the former is introduced as a central part of the latter. Shortly afterwards, in the April 1908 issue of The Monist, Peirce publishes the 'Amazing Mazes' in whose 'First curiosity' he defines 'theoric' in complete parallel to the usual definitions of 'theorematic' contrasting 'corollarial':

I shall term the step of so introducing into a demonstration a new idea not explicitly or directly contained in the premisses of the reasoning or in the condition of the proposition which gets proved by the aid of this introduction, a theoric step. (CP 4.613).

So, I just take 'theoric' to be another example of Peirce's proliferating neologisms where the same concept gives rise to the coining of many, dif- 
ferent terminological expressions for that concept. Hoffmann, on the other hand, remains right in pointing to the fact that Peirce's analysis of the recurrent example of Desargues' theorem does not coincide with his other examples and descriptions of theorematic reasoning - I would say it adds a further aspect to the description of varieties of theorematic reasoning. Adding a third dimension to the diagram of Desargues' theorem is adding a new element in another way than adding a particular line to an Euclidean diagram, because it induces a 'transformation' in the whole way of viewing the problem. When returning to Desargues' theorem the next year, in a letter to William James in 1909 (L224, NEM III, p. 47 1), Peirce now characterizes the Desargues proof as 'theorematic' and the introduction of a third dimension as yet another example of 'additions to the diagram.' The 'theoric' examples thus rather point to the fact that the range of possible additions and experiments in theorematical reasoning is fairly large, involving elements of highly different dimensionality, generality, and abstractness.

To sum up Peirce's different descriptions of theorematic reasoning, we can say they exceed the mere explication from the combination of definitions by introducing something further, be it new elements (particular or general), be it experiments by diagram manipulation, be it the substitution of schemata for words, or be it the gestalt shift of seeing the whole problem from another point of view. ${ }^{10}$

\section{Theorematical reasoning, relative to psychology? -or to logic systems chosen?}

But why could we not see the differences between corollarial reasoning and the different types of theorematic reasoning as a difference in reasoning psychology only? The former reasonings are generally taken to be easy while the latter require ingenuity - could we not reduce the difference between them to a difference between psychological resources needed to solve the problems? When only we are taught which lines to select in the angle sum proof or how to introduce the third dimension in Desargues' proof, these proofs become just as easy to conduct as corollarial proofs. The corollarial/ theorematical distinction would thus be reduced to one of psychology of learning, of the peculiarity of human reasoning capabilities to which some problems appear easy and other appear more difficult? Peirce, always alien 
to psychologism in logic, does not consider this possibility and maintains the idea that it is the very structure of the problem and the formal resources for its proof itself which gives rise to the distinction. ${ }^{11}$ Here, we take Peirce's stance in assuming that the difference in problem complexity is no purely psychological phenomenon.

A related idea rests on the fact that proofs of the same theorem may take many different forms - cf. the Desargues example — and so a logical parallel to such psychological ideas will be the following question: After a successful theorematic proof, could we not simply add the theorematic additions to the original set of premisses, the original statement of the problem - then the ensuing proof would become corollarial only and easy to perform? From time to time, Peirce toyed with the idea that proofs once having been theorematical might be transformed into the simpler form of corollarial reasoning by the change of logical system: 'Perhaps when any branch of mathematics is worked up into its most perfect form all its theorems will be converted into corollaries.' ('Sketch of Dichotomic Mathematics', c. 1903?, NEM III, p. 289). That corollarial proofs must be preferred to theorematic proofs for the same theorems, if available, follows from the obvious ideal that simpler proofs must be preferred to more complicated proofs of the same theorem - but this ideal does not grant that all of the latter may, in fact, be translated into the former. And even if some theorematical proofs may be translated into corollarial proofs, Peirce generally finds such an idea-not so far from a positive solution to Hilbert's Entscheidungsproblem -impossible, as we already saw in the 1902 quote where he deemed it impossible '... to define number in such a way that Fermat's or Wilson's theorems should be simple corollaries from the definition.' ('On Science and Natural Classes', 1902, EPII, p. 129).

At one occasion, in the 'Amazing Mazes', Peirce clearly distinguished between theorematic reasoning as such and the repetition of an already established theorematic proof, as well as between proofs necessarily requiring theorematic tools and theorematic proofs reducible to corollaries. (As in the 'Amazing Mazes' in general, Peirce here uses 'theoric' for 'theorematic'). A theorematic proof which may - if possible - be transformed into a simpler corollarial proof caused by the introduction of a better formal representation system, is called a 'theorem-corollary'-somewhat a misnomer. The repetition of a theorematic proof, once it has become familiar, 'a matter of course', and thus as easy as corollarial reasoning, he terms 'theoremation'- this must, 
of course, be distinguished from the former by still possessing the theorematic structure. Finally, the theorematic introduction of the new element in order to establish the proof is, by contrast, named a "major theorem":

Now to propositions which can only be proved by the aid of theoric steps (or which, at any rate, could hardly otherwise be proved), I propose to restrict the application of the hitherto vague word "theorem," calling all others, which are deducible from their premisses by the general principles of logic, by the name of corollaries. A theorem, in this sense, once it is proved, almost invariably clears the way to the corollarial or easy theorematic proof of other propositions whose demonstrations had before been beyond the powers of the mathematicians. That is the first secondary advantage of a theoric step. The other such advantage is that when a theoric step has once been invented, it may be imitated, and its analogues applied in proving other propositions. This consideration suggests the propriety of distinguishing between varieties of theorems, although the distinctions cannot be sharply drawn. Moreover, a theorem may pass over into the class of corollaries, in consequence of an improvement in the system of logic. In that case, its new title may be appended to its old one, and it may be called a theorem-corollary. There are several such, pointed out by De Morgan, among the theorems of Euclid, to whom they were theorems and are reckoned as such, though to a modern exact logician they are only corollaries. If a proposition requires, indeed, for its demonstration, a theoric step, but only one of a familiar kind, that has become quite a matter of course, it may be called a theoremation. If the needed theoric step is a novel one, the proposition which employs it most fully may be termed a major theorem; for even if it does not, as yet, appear particularly important, it is likely eventually to prove so. If the theoric invention is susceptible of wide. ('Amazing Mazes', 1908, CP 4.613)

The terminology of these distinctions seems not particularly well-chosen, one referring to the process ('theoremation') two to the result ('theorem-corollary', 'major theorem'), and the syncretistic notion 'theorem-corollary' doesn't seem to be a good means to indicate that the proposition in question is a corollary of one set of axioms, but not of another. The overall conceptual argument, however, clearly establishes the distinction between theorematic reasoning as such on the one hand, the issue of its dependency upon axiom and rule systems on other hand - and, finally, the psychological issue of its becoming familiar with repetition. As Hintikka $(1983,112)$ argues, the fact that some 
theorematical proofs become corollarial under other rule systems does not at all obliterate the corollarial-theorematical distinction, rather it makes clear that the distinction is relative to the logic system used and will remain, albeit differently, in any such system.

\section{Types of theorematic diagram experiments}

As we have seen, Peirce developed the distinction of corollarial and theorematical diagram deductions during the last 15 years of his life, and both explicitly and implicitly, he proposed different subtypes of theorematical deductions. He explicitly proposed a distinction between theorematic reasoning with or without abstractions, and more implicitly, distinctions may be inferred from his examples: manipulating with the diagram versus adding new material; the new elements added being objects, foreign ideas or new points of view. Apparently, he realized that all this laid out a whole field for further investigation:

I wish a historical study were made of all the remarkable theoric steps and noticeable classes of theoric steps. I do not mean a mere narrative, but a critical examination of just what and of what mode the logical efficacy of the different steps has been. Then, upon this work as a foundation, should be erected a logical classification of theoric steps; and this should be crowned with a new methodeutic of necessary reasoning. ('Amazing Mazes', 1908, CP 4.615)

The ultimate goal for such a research, as so much in Peirce, is heuristic ('methodeutic'): it should be undertaken in order to find better methods for deduction within the confines of the research process as such. The plurality of theorematic subtypes involved, already more or less vaguely glimpsed, may be no wonder, given the basic negative definition of somehow transgressing the merely definition-based corollarial reasoning. In how many ways is it possible to transgress corollarial reasoning? Given Peirce's overall continuism, we might surmise that these different subtypes of theorematic reasoning rather form a continuum from the simplest corollarial examples in the one end to the most complicated theorematical specimens in the other end. Hintikka proposed one arithmetic measuring stick for such a gradual scale - the amount of additional individuals needed in the proof. But this only goes for one subtype of 
theorematic reasoning, that of the introduction of further quantified particulars.

Among the many species of theorematical reasoning to investigate, we shall propose three logical levels of theorematical diagram experiment. Let us go back to the simple Euclidean example with the angle sum proof. Here, the introduction of auxiliary lines gives a basic example of the introduction of new particular objects. They are not in any way extraordinary - their very possibility is granted by basic Euclidean axioms and postulates. The only extraordinary thing about them is, as Shin argues, the selection of the right lines among the infinity of many possible.

A higher level of diagram experiment addresses the change of selected details of the very formalism making the former experiment possible. The famous geometrical example, of course, is the change of the parallel axiom ${ }^{12}$ which made possible the angle sum proof. This axiom was originally changed in order to try to find an ad absurdum proof: if a changed parallel axiom lead to inconsistencies in geometry, this would prove the parallel axiom was a theorem of the theory, and the rather cumbersome postulate could change status and become a theorem of geometry rather than part of the premisses. Famously, these attempts failed and lead, instead, to new systems of nonEuclidean geometries by Bolyai, Lobachevsky, Riemann, etc. in the mid-19th century. The parallel axiom could be changed, now, in two basic directions: instead of one possible parallel, given a line and a point, no parallel lines could be drawn through the point, or an infinity of parallel lines could be drawn - resulting in elliptic and hyperbolic geometries, respectively. But the very change of the parallel axiom is obviously an experiment with a wholly different status than the addition of auxiliary lines in the angle sum proof. Here, the very definition of which objects are taken to be possible in the formalism is changed - and a theorem such as the angle sum theorem will consequently be revised - in the former case, the angle sum will be more than 180 degrees, in the latter, less. Generally, experiments varying axioms, postulates, object definitions, transformation rules etc. of a theory must be ascribed a status different from the mere introduction of an object allowed by the existing rule system.

A still higher level, now, may be grasped from the same example. After the realization that three different types of geometries may result from the change of the parallel axiom, an experiment on an even higher level was possible: to vary and synthesize all such geometries and organize them on one continuum so that Euclidean geometry now forms a point with zero curvature of space 
on a line with different elliptic geometries having different positive curvatures, hyperbolic geometries having different negative curvatures (Bolyai, Riemann). By doing so, those pioneers undertook a step characterized by Peirce before he discovered the corollarial-theorematic distinction:

Mathematical reasoning consists in thinking how things already remarked may be conceived as making a part of a hitherto unremarked system, especially by means of the introduction of the hypothesis of continuity where no continuity had hitherto been thought of. ('Review of Spinoza's Ethic' 1894, in: Peirce, 1975-1987, II, pp. 84-85)

This third step realizes how Euclidean geometry and the infinite number of elliptic and hyperbolic geometries form part of 'a hitherto unremarked system' given by variation of curvature - exactly by taking them to be connected by the continuous variation of curvature.

Of course, still higher syntheses are possible - in geometry, the generalizations of the Erlangen program, defining different geometries by which invariances their transformation procedures allow for (thus finding a higher-order unremarked system of which both (non-)Euclidean geometries, projective geometry, and topology form a part) - or the generalization by Hilbert, taking the axiomatic structure of geometries as fixed while the interpretation of which model of objects they refer to could be subject to variation-or the generalization of category theory allowing for the co-articulation of geometry with different branches of mathematics, etc.

Such syntheses, however, seem to repeat the two latter types of theorematic experiments on higher levels. Thus, the three theorematic levels distinguished here - the introduction of a new object, and the two types of introducing a foreign idea, the experiment with one or more of the basic object or rule definitions, and the establishment of a system of different versions of those definitions, seem to to give us a hypothesis of three different types of theorematic diagram experiment.

\section{The three levels in applied diagrams}

Let us argue by example in discussing diagram experiments of these three kinds in applied diagrams, taking the geographical example of topographical 
maps. Here, the tracing of a route on the map from one location to another must constitute an example of corollarial reasoning. It does introduce new elements - the real or imaginary drawing of a line on the map, respecting, in addition to the mathematical aspects of the diagram, additional features of physico-geographical ontology: the trajectory should follow roads, not cross lakes, swamps, buildings, mountains etc. Geography, of course, is no fully axiomatized science, and the regional ontology of geography makes the additional geographical diagram constraints more vague than the exact mathematical aspects of the same diagram. A practical example of corollarial map reasoning may be the Danish police detective Jørn 'Old Man' Holm’s computer program, immediately plotting huge amounts of cell phone information related to suspects on a map:

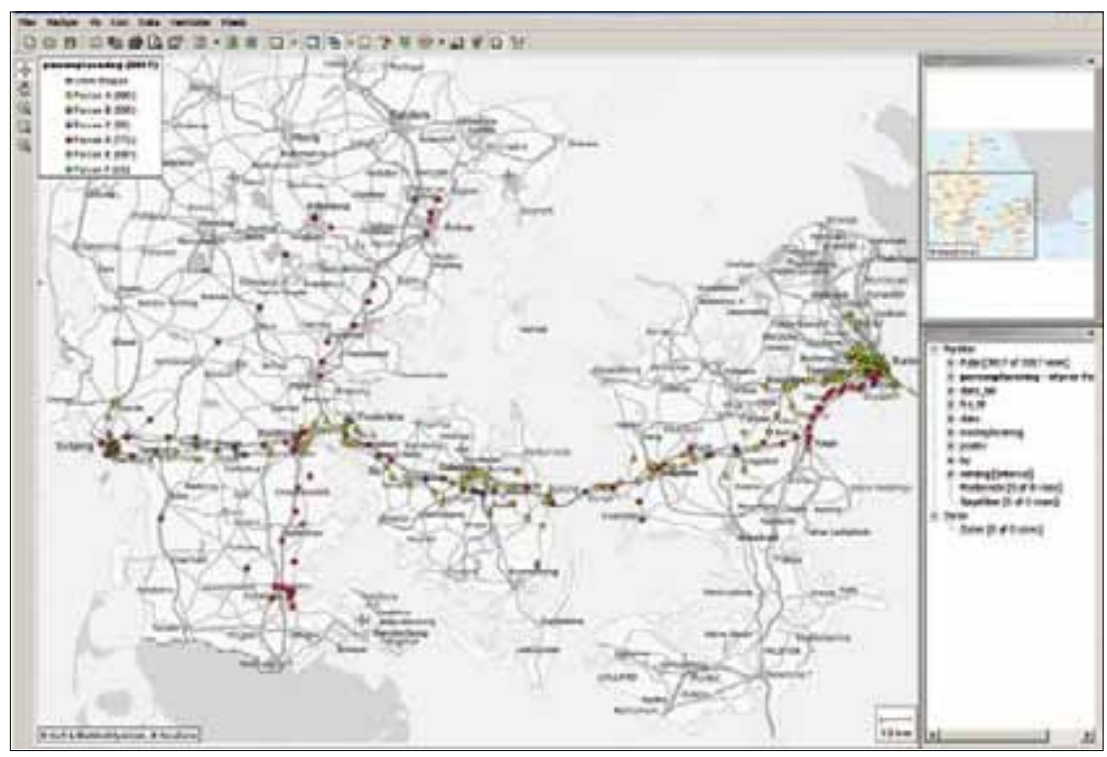

Fig. 4

Calls from the same cell phone are marked by the same colour. This diagram representation does not add anything new to previously existing information - except for the synthesizing a lot of single informations on one map, information which otherwise would have to be gathered from long lists of single pieces of longitude-latitude information of cell phone masts, cell phone 
numbers and call-up times. The synthesis of such information on one and the same diagram makes it possible to grasp in one glance gestalt information about cell phone trajectories on the map which would otherwise require complicated, time-demanding and not immediately convincing argumentation in court. Diagrammatic argumentation, by contrast, proves highly efficient in court where Holm has been called as an expert witness in many severe cases about drug smuggling, trafficking etc. Obviously, it becomes harder for a defendant to stay with his explanation that he spent the whole day in front of his tv set when a diagram proves that his cell-phone travelled from one end of the country and back the same day. Such information synthesis on a diagram constitutes an example of corollarial reasoning - unproblematically adding to the mathematical diagram aspects of points and lines those of the regional ontologies of geography and human communication.

Now, we may argue that the introduction of a new object in the diagram, e. g. in the shape of a ruler, marks a first small step in theorematical reasoning. It permits us to compare distances across the map - and even if having become an everyday utensil in our time and automatized in GPS and elsewhere, the ruler must have been a major breakthrough when the first distance calculation on a map was actually performed.

Still higher species of diagram experiment with maps may be gathered from science. A recent such example stems from Jared Diamond's celebrated book Guns, Germs, and Steel, tracing the roots of domesticated agriculture on Earth since the beginning of the neolithic period. A basic argument in the book comes from Diamond's diagram experiment with a world map (Diamond 2005, 177):

Fig. 5

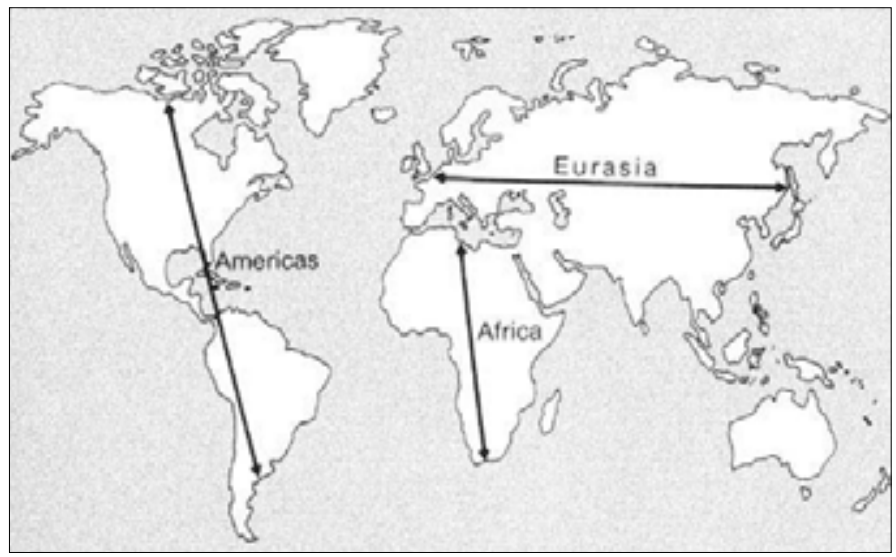


Diamond's basic observation is that among the three major continental complexes, Eurasia, Africa, and the Americas, there is a seminal differencethe former is roughly oriented East-West, while the latter are both oriented North-South. This almost trivial diagram experiment receives its non-triviality (which qualifies it as a piece of theorematic reasoning) from the underlying combination of biogeography with human culture in the regional ontology of the diagram. The domestication of plants and animals is a watershed event in human culture giving rise to the agricultural revolution and the development of large-scale, layered societies. Domestication presupposes the presence of easily domesticated species and the stable human settlement over many generations in the environment favoring the survival of these species. But local domestications only get the ability to dominate the development of human civilization if they are able to spread from there to other areas and cultures. But biogeographically, species are tied to local climate,- and local climate roughly depends on the latitude, forming isotherms across a temperature gradient falling from Equator towards the Poles. So the piecing together of these pieces of geographical ontology into a system depends upon a diagram experiment: once you have domesticated a species, where may it spread? Most favourably it spreads in the overall East-West direction, along isotherms, keeping climate conditions approximately constant - as opposed to traveling in the North-South direction where climate changes drastically with latitude. By this piece of a priori diagram reasoning - based on the combination of biogeographical ontology and the ontology of human culture developmentEurasia stands out as a privileged site for the original domestication of agricultural species (as opposed to Africa which might immediately be taken as a better candiate, original cradle of the human species as it is). Empirical findings subsequently corroborate this piece of theorematic reasoning: the fertile crescent of present-day Israel, Palestine, Syria, and Iraq seems to form the origin of many of the most important domestic species of the whole world, while the Far East comes in second. These areas were able to communicate domestications along the East-West axis and export them to Europe and the Far East. Of course, theorematicity must be a vaguer concept in empirical examples like this, where the ontologies of biogeography and of culture are not exhaustively described, but still an argument may be made which runs as follows. The complex of three basic propositions 1) domestication of a species is a local event, 2) requiring stable human settlement in the natural niche of the species 3) and the spread of such species must favor isotherms, 
combined with the diagram experiment of searching the world map where the most favorable isotherms occur, constitutes a piece of theorematic reasoning. It introduces a new object on the map - the possible spreading trajectories of domesticated animal and plant species - and thus hypothesizes a general regularity on the globe. This experiment on the map involves the combination of concepts from different regional ontologies, of geography, biogeography, meteorology, cultural history - in some sense, it synthesizes different world maps charting findings in these different disciplines as a prerequisite of the experiment. Thus, it provides a new argument for which cultures were able to survive. But it does not introduce a foreign idea.

A further geographical example may be the more famous diagram experiment by the German geographer and explorer Alfred Wegener leading to the plate-tectonics of current geology. Famously, Wegener was toying with a map and noticing that the West coast of Africa strangely fit like a puzzle piece into the East coast of South America:

Fig. 6

The coastline fit of South America and Africa, supplemented with geological similarities, from Wegener 1929, p. 73 (the illustration adapted by Wegener from Alexander du Toit).

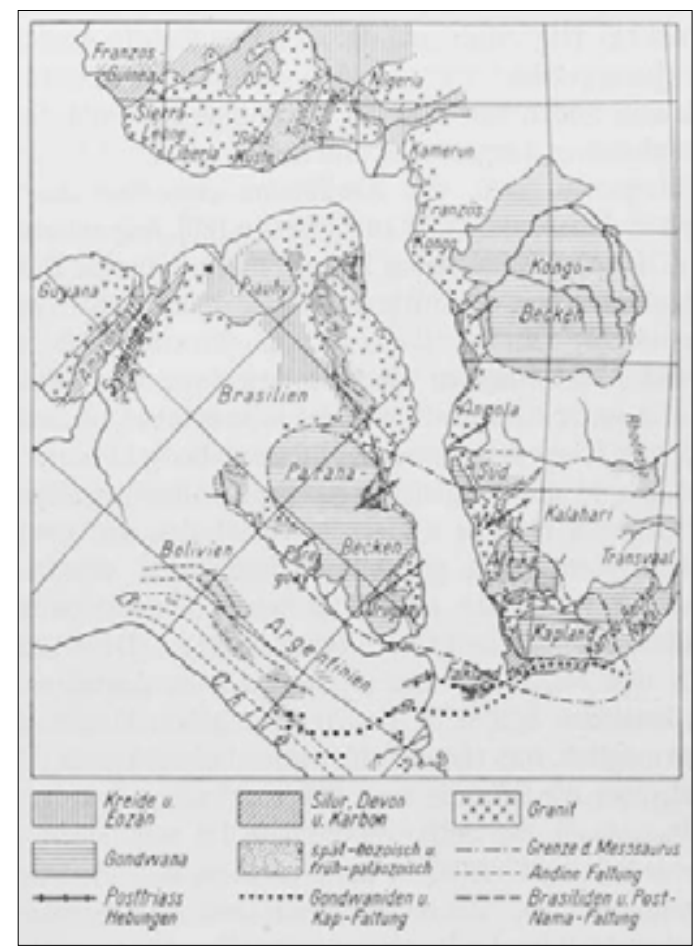


This lead to Wegener's groundbreaking 1912 idea ('Die Entstehung der Kontinente') that these continents had once been one-a controversial argument initially ridiculed, but much later corroborated by the findings of geological and biological similarities along the two coasts and finally accepted after the discovery of the mid-Atlantic mountain range as the decisive indication that the ocean does in fact 'grow' in the middle. This diagram experiment belongs to a second level as compared to the Jared Diamond example. Here, not only new objects or connections are introduced-here a completely new idea is introduced, namely that of continents moving over time. Taken on the level of pure diagrams, of course, nothing is strange - all Wegener did was to take a geometrical object and make a classical rigid Euclidean movement in order

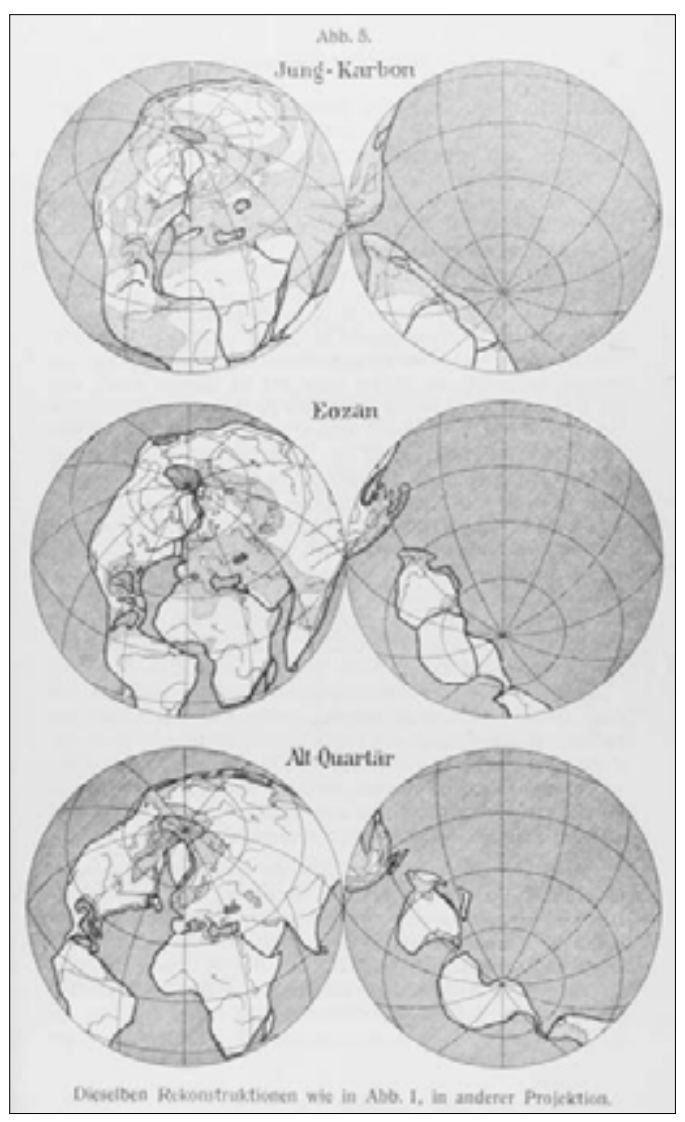

Fig. 7

Reconstructions of the world map accordig to the continental drift theory, from Wegener 1929, p. 19 
to let it face another object. This is permitted by geometry, but, of course, not by the regional ontology of pre-Wegener geography. So Wegener's diagram experiment changed an axiom of geography, as it were-the assumption of long-term stability of large-scale features of the Earth surface - and so introduced not only a new object, but a foreign idea, that of continents moving on a geological timescale.

An example of a third level diagram experiment in geography might be taken from the same piece of history of science: the reinterpretation of the whole of the surface of the Earth in terms of moving continental plates, inverting their present movements and extrapolating them into the past in order to trace the origins of the continents. Mountain ranges were now seen as the results of continent collisions and volcanic areas as the result of chasms between plates going in each their direction. This permitted the coordinated diagram experiment reconstructing the original ur-continent of Pangaea. Wegener already presented the idea of the ur-continent in his 1915 book, it was baptized Pangaea at at 1928 conference, and was presented like this in the 1929 version of his book Die Entstehung der Kontinente und Ozeane shortly before his death in 1930 (see Fig. 7).

Here, the particular change of an axiom lead to the systematic reinterpretation of a whole conceptual structure of geographical and geological ontology, effectively integrating the two into one discipline by seeing the same forces in work all over the surface of the Earth - in some ways comparable to the systematization of geometries after degrees of curvature.

\section{Theorematic reasoning and Hypostatic Abstraction}

We may sum up our hypothesis of three basic levels of Theorematic reasoning as follows:

1) Addition of new individuals to the premises

2) Higher-level experiment with variation of axiom or transformation rule

3) Establishment of system of different axioms or rules

Now, what is the relations of them to Hypostatic Abstraction - the procedure Peirce described as making a second-level substantive out of a first-level predicate, thereby creating a new object of thought? As we have seen in this 
paper, Peirce sometimes distinguishes non-abstract from abstract theorematic reasonings; while in an early quote he almost identifies abstraction with theorematic reasoning. Immediately, the talk about individual instantiation in the first theorematic reasoning type seems to preclude that abstraction should play any role here. Peirce's debatable analysis of lines as abstractions from the trajectories of moving particles would make the subsidiary lines in the angle sum proof abstract objects added in the proof. In any case, the importance of this step lies in the selection of the individual lines needed for the proof which is not a matter of abstraction. Likewise, such lines do not add an idea which could be said to be foreign to the theorem to be proved. Maybe the first-level addition of new individuals could comprise both abstract and non-abstract cases.

Different, however, seems the case of the second level of theorematic reasoning, implying that something in the rule system is taken as object of experiment, leading to a 'foreign idea'. If the parallel axiom is what defines the hypostatic abstraction of 'parallelness' or 'being parallel', then the variation of that axiom introduces competing definitions of that abstraction - the 'foreign ideas' of that level.

The third level, then, would be that of making a whole system out of hypostatic abstractions - this system constituting itself, then, a complex hypostatic abstraction on a higher level, involving such new hypostatic terms as 'curvature of space'. This level seems characterized by 'the introduction of the hypothesis of continuity where no continuity had hitherto been thought of', as Peirce had it - establishing continua of hypostatic abstractions from the second level.

No doubt, the relation between theorematic reasoning and abstraction allows for many sophistications which is beyond our scope to investigate here. This must be left for further reasoning.

Thanks to Michael Hoffmann, Ahti Pietarinen, Sun-Joo Shin, and André de Tienne for comments and help. 


\section{Notes}

1 Taking Comte's principle (a science is below a another science from which it takes its principles, and above another science whose principles borrows from it) as basis for his classifications of the sciences, Peirce places mathematics on top as the science from which all other sciences borrow principles.

2 These equivalences between alternate angles, of course, are granted by Euclid's Proposition 29: If two straight lines are parallel, then a straight line that meets them makes the alternate angles equal.

3 Shin's investigation of syntheticity in Kant and Peirce concludes as follows (1997, 37): 'Even though Peirce himself did not engage in a full evaluation of Kant's analytic/synthetic distinction, we can now acquire a quite comprehensive picture of this matter. Peirce's theory of mathematics locates the origin of Kant's syntheticity in two different sources: One is the historical fact that Kant was writing before the discovery of quantification theory, and the other is Kant's appreciation of different kinds of reasoning. The former source is responsible for Kant's claim about the statement " $7+5=12$ ", but his claim about the statement. "A triangle's angles sum to 180 degrees" is related to the latter. The development of logic cleared away the first source of syntheticity, and we know that this origin of Kant's syntheticity is not sustainable any more.' Consequently, the other, more sound aspect of Kant's syntheticity relies in his appreciation of two different modes of reasoning, logical and mathematical, - which were reconstructed by Peirce as corrolarial and theorematical reasoning, respectively. See also Stjernfelt 2007, ch. 8.

4 As abstractions come in many levels, and abstract/concrete is not coextensive with general/particular, the issue whether the subsidiary lines should be taken as abstractions or particulars or both needs not bother us.

5 The selection of the right elements to add is abductive. In the angle sum case, the addition of parallel lines is probably prompted by the previous knowledge of Proposition 29 dealing with the relation between parallel lines and the size of alternate angles - because the theorem to be proved is about angle sizes. So even if the selection itself is not deductive but merely abductive, the abduction is motivated by a certain likeness between the theorem and possibilities offered by previous theorems proved.

6 A parallel quote, emphazising the theorematic step as the addition of a new idea, is the following: 'I shall term the step of so introducing into a demonstration a new idea not explicitly or directly contained in the premisses of the reasoning or in the condition of the proposition which gets proved by the aid of this introduction, a 
theoric step.' ('Amazing Mazes', 1908, CP 4.613)

7 The shortest presentation is probably: 'For mathematical reasoning consists in constructing a diagram according to a general precept, in observing certain relations between parts of that diagram not explicitly required by the precept, showing that these relations will hold for all such diagrams, and in formulating this conclusion in general terms. All valid necessary reasoning is in fact thus diagrammatic.' ('Lessons from the History of Science', 1896, CP 1.54) The object of mathematics will be pure diagrams of any kind, while ordinary reasoning as well as the empirical sciences will use diagrams applied in being constrained by existing relationsempirical data and regional ontology - as well.

8 A detailed attempt at developing the distinction between corollarial and theorematical reasoning in 'The Logic of History' (1901, NEM IV) takes the proof of $(\mathrm{x}+\mathrm{y})+\mathrm{z}=\mathrm{x}+(\mathrm{y}+\mathrm{z})$ as example of the former and the proof that 'every multitude is less than a multitude' ( $\approx$ there is no largest set) as example of the latter. The overall argument, however, is not very clear. Especially the latter proof which seems to be a sort of diagonal argument implying a power set construction is not very clearly presented, and even if Peirce concludes it 'requires the invention of an idea not at all forced upon us by the terms of the thesis', he does not make explicit what that new idea should be, apart from not being derivable from the definition of the concept 'fewer' (a clearer example of Peirce's version of Cantor's power set theorem can be found a few years earlier in 'The Logic of Relatives' (1897, CP 3.548). Similarly, at the end of 'Logic of History', Peirce has a brief and clear summary of the Power Set Theorem: 'I proved that there is no maximum multitude by considering the collection of all possible collections of the numbers of a collection. Now a collection is an abstraction ...' (1901, NEM IV, p. 11), but still there is no indication of what the 'new idea' introduced should be. Certainly not the abstraction of 'collection' which is presupposed by the multitudes of the premisses - rather the power set notion of the set of all possible subsets of a given set. Peirce seems to have realized the early discussion of corollarial/theorematical in 'The Logic of History' was less than satisfying; according to the Robin catalogue on the Ms. (691), Peirce added the following note to the Ms.: 'These pages are to be used in the chapter of the Logic treating Deductive Reasoning. But the theory needs completion.'

9 Here, I quote Ms. 754 from Hoffmann (forthcoming) as the ISP page 8 is missing from the Microfilm edition where it seems to belong between pages 5 and 6 .

10 Maybe the very concept of theorematical reasoning is necessarily open - given the inexhaustibility of mathematics, it may not be granted we should be able to chart 
all possible subtypes of such reasoning beforehand?

11 A related issue is the degree of conscious access to reasoning processes: 'If, however, as the English suppose, the feeling of rationality is the product of a sort of subconscious reasoning - by which I mean an operation which would be a reasoning if it were fully conscious and deliberate - the accompanying feeling of evidence may well be due to a dim recollection of the experimentation with diagrams.' (CP 2.172) The experience of evidence resulting from rational reasoning may, in some cases, depend upon subconscious reasoning. A recurring argument in Peirce, however, points to the fact that such reasoning - and, even more, that of computers-lacks self-control as the hallmark of real reasoning. As to mechanization of reasoning, Peirce often discusses the corollarial/theorematical distinction with reference to computers ('logic machines'). His overall idea is that the former will generally be mechanizable, while the latter lie beyond mechanization because their introduction of new elements by experiment requires creativity and ingenuity. These ideas might be seen as a vague anticipation of later discoveries of decision limitations in the philosophy of mathematics and computation (Gödel's incompleteness theorem, or Turing's related halting problem), but they are not simply equivalent. These limitations have another character than Peirce's distinction, because they limit the range even of purely mechanical decision procedures which Peirce would, in all probability, categorize as corollarial.

12 Strictly speaking, the parallel postulate. It has become common usage to call it an axiom.

\section{Literature}

Benacerraf, P. with H. Putnam (eds.) 1983 Philosophy of Mathematics, Cambridge Mass.: Cambridge University Press.

Brunning, Jacqueline and Forster, Paul (eds.) 1997 The Rule of Reason. The Philosophy of Charles Sanders Peirce, Toronto: University of Toronto Press.

Cooke, E. 2010 'Peirce's General Theory of Inquiry and the Problem of Mathematics', in Moore 2010, 169-292.

Diamond, Jared 2005 Guns, Germs and Steel, London: Vintage, 2005 [1997].

Dipert, Randall 1995 'Peirce's Underestimated Place in the History of Logic: A Response to Quine', in Ketner 1995, 32-58.

— 1997 'Peirce's Philosophical Conception of Sets', in Houser et al. (eds.) 1997.

- 2004 'Peirce's Deductive Logic: Its Development, Influence, and Philosophical 
Significance', in Misak 2004, 287-324.

Dougherty, Charles J. 1983 'Peirce's Phenomenological Defense of Deduction', in Freeman 1983, 167-177.

Fisch, Max 1986 'Peirce’s Progress from Nominalism Towards Realism', in Fisch (eds.

K. L. Ketner and C.J. W. Kloesel) Peirce, Semeiotic, and Pragmatism, Bloomington: Indiana University Press, 184-200.

Freeman, Eugene (ed.) 1983 The Relevance of Charles Peirce, La Salle, Illinois 1983: The Hegeler Institute.

Hintikka, Jaakko 1983 'C. S. Peirce's "First Real Discovery” and Its Contemporary Relevance', in E. Freeman (ed.) The Relevance of Charles Peirce, La Salle, Ill. 1983: The Hegeler Institute, 107-118.

— 1997 'The Place of C. S. Peirce in the History of Logical Theory', in J. Brunning and P. Forster (eds.) The Rule of Reason, Toronto etc.: University of Toronto Press 1997, 13-33 (also in Hintikka 1997, p. 140-61).

- 1997a Lingua Universalis vs. Calculus Ratiocinator. An Ultimate Presupposition of Twentieth-Century Philosophy, Dordrecht etc. 1997: Kluwer.

Hoffmann, Michael 2005 Erkenntnisentwicklung. Ein semiotisch-pragmatischer Ansatz, Frankfurt: Klostermann.

— 2006 'Seeing Problems, Seeing Solutions. Abduction and Diagrammatic Reasoning in a theory of Scientific Discovery', Working Paper \#2006.15, Georgia Institute of Technology.

— forthcoming "Theoric Transformations" and a New Classification of Abductive Inferences', in: Transactions of the Charles S. Peirce Society.

Houser, Nathan, Don D. Roberts and James van Evra (eds.) 1997 Studies in the Logic of Charles Sanders Peirce, Bloomington: Indiana University Press.

Levy, Stephen H. 1997 'Peirce's Theoremic/Corollarial Distinction and the Interconnections between Mathematics and Logic', in Houser et al. (1997), p. 85-110.

Moore, Matthew E. 2010 (ed.) New Essays on Peirce's Mathematical Philosophy, Chicago and La Salle: Open Court.

Peirce, Charles S. 1976 New Elements of Mathematics, (ed. C. Eisele) I-IV, The Hague: Mouton.

- 1975-87 Contributions to the Nation, I-IV (ed. K. L. Ketner \& J. E. Cook), Lubbock TX: Texas Technological University Press.

- 1992 Reasoning and the Logic of Things, (eds. Ketner and Putnam), Camb.Mass.: Harvard University Press.

— 1992 The Essential Peirce, vol. I. (1867-1893), [EPI], eds. N. Houser and C. Kloesel, Bloomington: Indiana University Press. 
- 1997 Pragmatism as a Principle, (ed. A. Turrisi), Albany: SUNY Press.

- 1998 Collected Papers [CP, references given by volume number and paragraph], I-VIII, (ed. Hartshorne and Weiss; Burks) London: Thoemmes Press (1931-58).

- 1998 The Essential Peirce, vol. II (1893-1913) [EPII], eds. N. Houser and C. Kloesel, Bloomington: Indiana University Press.

- 2010 Philosophy of Mathematics. Selected Writings, ed. M. Moore, Bloomington and Indianapolis: Indiana University Press.

- (no year) 'Logic, Considered as Semeiotic' [LCS], constructed from manuscript L 75 by Joseph Ransdell (1902) (http://www.cspeirce.com/menu/library/bycsp/ 175/ver1/175v1-01.htm).

- Manuscripts at the Houghton Library referred to by Ms. numbers in the Microfilm edition 1966 The Charles S. Peirce Papers, Microfilm Edition, Thirty Reels with Two Supplementary Reels Later Added. Cambridge: Harvard University Library Photographic Service. Manuscript numbers also refer to the Robin catalogue of the Mss. (Robin 1967). As to manuscript page numbers, 'CSP' refers to Peirce's pagination (which is not unanimous since several parallel drafts may belong to the same Ms.), while 'ISP' refers to the pagination of the Mss. by the Institute for Studies in Pragmaticism, also used by the Peirce Edition Project in Indianapolis. When ISP numbers are not available, numbers refer to the sequence of the microfilm reel, e. g. 'Reel 16, 1434-35' meaning the 1434th and 1435th page of reel 16 .

Robin, Richard 1967 Annotated Catalogue of the Papers of Charles S. Peirce, Worcester Mass.: University of Massachusetts Press 1967.

Shin, Sun-Joo 1994 The Logical Status of Diagrams, Cambridge: Cambridge University Press.

— 1997 'Kant's Syntheticity Revised by Peirce', Synthese 113, 1-41.

- 2000 The Iconic Logic of Peirce's Graphs, Cambridge MA: MIT.

— 2010 'Peirce's Two Ways of Abstraction', in Moore 2010, 41-58.

Stjernfelt, Frederik 2007 Diagrammatology. An Investigation on the Borderlines of Phenomenology, Ontology, and Semiotics, Dordrecht: Springer Verlag.

- forthcoming a) 'Signs Conveying Information. On the range of Peirce's notion of propositions: Dicisigns', in Fournal of Signs and Semiotic Systems.

- forthcoming b) 'The evolution of semiotic self-control. Sign evolution as the ongoing subdivision, articulation and making explict of a basic metabolic argument structure', in T. Schilhab, F. Stjernfelt, and T. Deacon (eds.) New Perspectives on the Symbolic Species, Yale University Press.

— forthcoming c) 'The Generality of Signs. Anti-Psychologism in the Foundations of Semiotics - and its Actual Relevance', in Semiotica. 
Tiercelin, Claudine 2010 'Peirce on Mathematical Objects and Mathematical Objectivity', in Moore 2010, 81-122.

Webb, Judson 2006 'Constructions, Intuitions, and Theorems', in Randall E. Auxier and Lewis Edwin Hahn (eds.) The Philosophy of Jaakko Hintikka, Chicago etc.: Open Court, p. 196-301.

Wegener, Alfred 1912 'Die Entstehung der Kontinente', Geologische Rundschau 3(4), 276-292.

- 1929 Die Entstehung der Kontinente und Ozeane [The Origin of Continents and Oceans] (4 ed.). Braunschweig: Friedrich Vieweg \& Sohn. 